\title{
A Comparative Study on Support Vector Machine and Constructive RBF Neural Network for Prediction of Success of Dental Implants
}

\author{
Adriano L.I. Oliveira ${ }^{1}$, Carolina Baldisserotto ${ }^{1}$, and Julio Baldisserotto ${ }^{2}$ \\ 1 Department of Computing Systems, Polytechnic School of Engineering, \\ Pernambuco State University, \\ Rua Benfica, 455, Madalena, Recife - PE, Brazil, 50.750-410 \\ 2 Faculdade de Odontologia, Universidade Federal do Rio Grande do Sul, \\ Rua Ramiro Barcelos, 2492, Porto Alegre - RS, Brazil, 90.040-060 \\ adriano@dsc.upe.br, carol_baldi@yahoo.com.br, bjulio@ghc.com.br
}

\begin{abstract}
The market demand for dental implants is growing at a significant pace. In practice, some dental implants do not succeed. Important questions in this regard concern whether machine learning techniques could be used to predict if an implant will be successful and which are the best techniques for this problem. This paper presents a comparative study on three machine learning techniques for prediction of success of dental implants. The techniques compared here are: (a) support vector machines (SVM); (b) weighted support vector machines; and (c) constructive RBF neural networks (RBF-DDA) with parameter selection. We present a number of simulations using real-world data. The simulations were carried out using 10-fold cross-validation and the results show that the methods achieve comparable performance, yet RBF-DDA had the advantage of building smaller classifiers.
\end{abstract}

\section{Introduction}

Dental implants have been used successfully to replace lost teeth with very high success rates $[3$. Nevertheless, oral rehabilitation through dental implants presents failure risks related to the different phases of the osseointegration process (the integration of the implant to the adjacent bone) [13]. A number of risk factors may be related to the failure of dental implants, such as the general health conditions of the patient, the surgical technique employed, the use of smoke by the patient and the type of implant 12 . In this work, a dental implant is considered successful if it presents characteristics of osseointegration in the different phases of the process, including the prosthetic loading and its preservation. We considered that a failure took place whenever any problem related to the implant motivated its removal.

The features of the patients considered in this work were carefully chosen by an oral surgeon specialist in dental implants. The features considered here were: 1) age of the patient, 2) gender, 3) implant type, 4) implant position, 5) surgical 
technique, 6) an indication whether the patient was a smoker of not and 7) an indication whether the patient had a previous illness (diabetes or osteoporosis) or medical treatment (radiotherapy). These features are best described in the remaining of the paper. Some of these features, also referred to as risk factors, were also considered in a recent studied which used statistical techniques to analyze the risk factors associated with dental implants [12. The data for the present study were collect between the years 1998 and 2004 by a single oral surgeon. The data set consists of 157 patterns which describe dental implants.

In the period in which data were collected there were implants carried out less than five years before. Therefore, instead of classifying the outcome of an implant simply as success or failure, we have classified our data into seven classes: (1) success confirmed until one year; (2) success confirmed between 1 and 2 years; (3) success confirmed between 2 and 3 years; (4) success confirmed between 3 and 4 years; (5) success confirmed between 4 and 5 years; (6) success confirmed for more than 5 years; and (6) failure. In general, the longer the number of years of confirmed success, the greater is the likelihood of definitive success of an implant.

Nowadays the prediction of success of failure of a dental implant is almost always carried out by the oral surgeons through clinical and radiological evaluation. Therefore, the accuracy of such predictions is heavily dependent on the experience of the oral surgeon. This works aims to help predicting the success or failure of a dental implant via machine learning techniques, thereby hoping to improve the accuracy of the predictions.

We have considered three machine learning techniques for our comparison, namely, (a) support vector machines (SMVs) [781]; (b) weighted SVMs [106]; and (c) RBF-DDA with $\theta^{-}$selection [17].

SVMs are a recent powerful class of machine learning techniques based on the principle of structural risk minimization (SRM). SVMs have been applied successfully to a wide range of problems such as text classification and optical character recognition [818. Weighted SVM is an extension to SVM more appropriate to handle imbalanced datasets, that is, datasets which have unequal proportion of samples between classes 106 . We have considered this extension to SVM here because our dataset is imbalanced, as detailed in section 3. DDA is a fast training method for RBF and PNN neural networks [5]. RBF-DDA with $\theta^{-}$selection uses cross-validation to select the value of parameter $\theta^{-}$thus improving performance in some classification problems [17]. We decided to use the last classifier in order to assess its performance in a task different from those considered in the paper in which it was originally proposed [17. Thus this paper also contributes by further exploring this classifier on a different data set.

The classifiers considered in this work were compared using 10-fold crossvalidation together with Student paired t-tests with $95 \%$ confidence level.

This paper is organized as follows. Next section reviews the machine learning techniques considered in this work. Section 3 describes the experiments carried out along with the results and discussion. Finally, section 4 presents our conclusions and suggestions for further research. 


\section{The Machine Learning Techniques Compared}

\subsection{Support Vector Machines}

Support vector machine (SVM) is a recent technique for classification and regression which has achieved remarkable accuracy in a number of important problems [71881. SVM is based on the principle of structural risk minimization (SRM), which states that, in order to achieve good generalization performance, a machine learning algorithm should attempt to minimize the structural risk instead of the empirical risk [81]. The empirical risk is the error in the training set, whereas the structural risk considers both the error in the training set and the complexity of the class of functions used to fit the data. Despite its popularity in the machine learning and pattern recognition communities, a recent study has shown that simpler methods, such as $\mathrm{kNN}$ and neural networks, can achieve performance comparable to or even better than SVMs in some classification and regression problems [14].

The main idea of support vector machines is to built optimal hyperplanes that is, hyperplanes that maximize the margin of separation of classes - in order to separate training patterns of different classes. An SVM minimizes the first equation below subject to the condition specified in the second equation

$$
\begin{aligned}
\min _{w, b, \xi} & \frac{1}{2} w^{T} w+C \sum_{i=1}^{l} \xi_{i} \\
\text { subject to } & y_{i}\left(w^{T} \phi\left(x_{i}\right)+b\right) \geq 1-\xi_{i}, \\
& \xi_{i} \geq 0 .
\end{aligned}
$$

The training vectors $x_{i}$ are mapped into a higher (maybe infinite) dimensional space by the function $\phi$. Then SVM finds a linear separating hyperplane with the maximal margin in this higher dimensional space. A kernel $K(\vec{x}, \vec{y})$ is an inner product in some feature space, $K(\vec{x}, \vec{y})=\phi^{T}(\vec{x}) \phi(\vec{y})$. A number of kernels have been proposed in the literature [18 812 . In this work we use the radial basis function ( $\mathrm{RBF}$ ) kernel, which is the kernel used more frequently. The kernel function $K\left(x_{i}, x_{j}\right)$ in an RBF kernel is given by $K\left(x_{i}, x_{j}\right)=\exp \left(-\gamma \| x_{i}-\right.$ $\left.x_{j} \|^{2}\right), \gamma>0$.

SVMs with RBF kernels have two parameters, namely, $C$, the penalty parameter of the error term $(C>0)$ and $\gamma$, the width of the RBF kernels. These parameters have great influence on performance and therefore their values must be carefully selected for a given problem. In this work, model selection is carried out via 10-fold cross-validation on training data. A grid search procedure on $C$ and $\gamma$ is performed, whereby pairs of $(C, \gamma)$ are tried and the one with the best cross-validation accuracy is selected [11. A practical method for identifying good parameters consists in trying exponentially growing sequences of $C$ and $\gamma$. In our experiments, the sequence used was $C=2^{-5}, 2^{-3}, \cdots, 2^{15}$, and $\gamma=2^{-15}, 2^{-13}, \cdots, 2^{3}[1]$. 


\subsection{Weighted Support Vector Machines}

Weighted support vector machine (WSVM) was proposed to address two important problems which appear quite often in pattern recognition, namely, (1) classification problems with imbalanced datasets, that is, datasets in which the classes are not equally represented; and (2) classification problems in which a classification error of one type is more expensive or undesirable than other [10].

The idea of WSVM consists in penalizing with higher penalty the most undesirables types of errors 1016. For this purpose, WSVMs have one weight $w_{i}$ per class. In WSVMs each class $i$ has a different penalty parameter $C_{i}$. This is in contrast to the original SVM, which has only one the penalty parameter of the error term $(C>0)$ (equation (1)), which is used for all classes. The parameter $C_{i}$ in WSVMs is set to $w_{i} C$. In practice, higher values of $w_{i}$ should be used for classes with smaller number of samples.

The motivation for considering WSVMs in our problem is that our dataset is imbalanced, in particular, we have quite few cases of failure of dental implants (as detailed in section 3).

\subsection{Constructive RBF Neural Networks}

The DDA algorithm is a very fast constructive training algorithm for RBF and probabilistic neural networks (PNNs) [5]4. In most problems training is finished in only four to five epochs. The algorithm has obtained good performance in a number of problems, which has motivated a number of extensions to the method recently proposed in the literature [17/16[15].

An RBF trained by DDA is referred as RBF-DDA. The number of units in the input layer represents the dimensionality of the input space. The input layer is fully connected to the hidden layer. RBF-DDAs have a single hidden layer. The number of hidden units is automatically determined during training. Hidden units use Gaussian activation functions. RBF-DDA uses 1-of-n coding in the output layer, with each unit of this layer representing a class. Classification uses a winner-takes-all approach, whereby the unit with the highest activation gives the class. Each hidden unit is connected to exactly one output unit. Each of these connections has a weight $A_{i}$. Output units uses linear activation functions with values computed by

$$
f(\vec{x})=\sum_{i=1}^{m} A_{i} \times R_{i}(\vec{x})
$$

where $m$ is the number of RBFs connected to that output.

The DDA training algorithm is constructive, starting with an empty hidden layer, with units being added to it as needed. The centers of RBFs, $\overrightarrow{r_{i}}$, and their widths, $\sigma_{i}$ are determined by DDA during training. The values of the weights of connections between hidden and output layers are also given by DDA.

The complete DDA algorithm for one training epoch is shown in Fig. 1. The algorithm is executed until no changes in the parameters values (number of 
hidden units and their respective parameters and weights values) are detected. This usually takes place in only four to five epochs [5]. This natural stopping criterion leads to networks that naturally avoid overfitting training data [54].

1: // reset weights:

FORALL prototypes $p_{i}^{k}$ DO

$$
A_{i}^{k}=0.0
$$

\section{ENDFOR}

2: // train one complete epoch

FORALL training pattern $(\vec{x}, c)$ DO

IF $\exists p_{i}^{c}: R_{i}^{c}(\vec{x}) \geq \theta^{+}$THEN

3: // sample covered by existing neuron of the same class $A_{i}^{c}+=1.0$

\section{ELSE}

4: // "commit": introduce new prototype add new prototype $p_{m_{c}+1}^{c}$ with:

$\vec{r}_{m_{c}+1}^{c}=\vec{x}$

$A_{m_{c}+1}^{c}=1.0$

$m_{c}+=1$

5: // adapt radii

$\sigma_{m_{c}+1}^{c}=\max _{k \neq c \wedge 1 \leq j \leq m_{k}}\left\{\sigma: R_{m_{c}+1}^{c}\left(\vec{r}_{j}^{k}\right)<\theta^{-}\right\}$

\section{ENDIF}

6: // "shrink": adjust conflicting prototypes

FORALL $k \neq c, 1 \leq j \leq m_{k}$ DO

$$
\sigma_{j}^{k}=\max \left\{\sigma: R_{j}^{k}(\vec{x})<\theta^{-}\right\}
$$

\section{ENDFOR}

\section{ENDFOR}

Fig. 1. DDA algorithm for one training epoch

The DDA algorithm relies on two parameters in order to decide about the introduction of new prototypes (RBF units) in the networks. One of these parameters is a positive threshold $\left(\theta^{+}\right)$, which must be overtaken by an activation of a prototype of the same class so that no new prototype is added. The other is a negative threshold $\left(\theta^{-}\right)$, which is the upper limit for the activation of conflicting classes [54].

A trained RBF-DDA network holds the following two equations for every training pattern $\vec{x}$ of class $c[5]$ :

$$
\begin{gathered}
\exists i: R_{i}^{c}(\vec{x}) \geq \theta^{+} \\
\forall k \neq c, 1 \leq j \leq m_{k}: R_{j}^{k}<\theta^{-}
\end{gathered}
$$

Notice that the above conditions do not guarantee the correct classification of all training patterns, because they hold for hidden units, not for output units.

During training, the DDA algorithm creates a new prototype for a given training pattern $\vec{x}$ only if there is no prototype of the same class in the network 
whose output $R_{i}(\vec{x}) \geq \theta^{+}$. Otherwise, the algorithm only increments the weight $A_{i}$ of the connection associated with one of the RBFs (of the same class of the training pattern) which gives $R_{i}(\vec{x}) \geq \theta^{+}$(step 3 of Fig. 1). When a new prototype is introduced in the network, its center will have the value of the training vector $\vec{x}$ and the weight of its connection to the output layer is set to 1 (step 4 of Fig. 1). The width of the Gaussian will be chosen in such a way that the outputs produced by the new prototype for existing prototypes of conflicting classes is smaller than $\theta^{-}$(step 5 of Fig. 1). Finally, there is a shrink phase, in which the widths of conflicting prototypes are adjusted to produce output values smaller than $\theta^{-}$for the training pattern $\vec{x}$ (step 6 of Fig. 1 ).

Originally, it was assumed that the value of DDA parameters would not influence classification performance and therefore the use of their default values, $\theta^{+}=0.4$ and $\theta^{-}=0.1$, was recommended for all datasets 54. In contrast, it was observed more recently that, for some datasets, the value of $\theta^{-}$considerably influences generalization performance in some problems [17. To take advantage of this observation, a method has been proposed for improving RBF-DDA by carefully selecting the value of $\theta^{-}$[17.

In the RBF-DDA with $\theta^{-}$selection method, the value of the parameter $\theta^{-}$is selected via cross-validation, starting with $\theta^{-}=0.1$ [17. Next, $\theta^{-}$is decreased by $\theta^{-}=\theta^{-} \times 10^{-1}$. This is done because it was observed that performance does not change significantly for intermediate values of $\theta^{-}$[17. $\theta^{-}$is decreased until the cross-validation error starts to increase, since smaller values lead to overfitting [17]. The near optimal $\theta^{-}$found by this procedure is subsequently used to train using the complete training set [17. The algorithm is shown in Fig. 2.

$\theta_{\text {opt }}^{-}=\theta^{-}=10^{-1}$

Train one RBF-DDA with $\theta^{-}$using the reduced training set and test on the validation set to obtain ValError $=$ MinValError

REPEAT

$\theta^{-}=\theta^{-} \times 10^{-1}$

Train one RBF-DDA with $\theta^{-}$using the reduced training set and test on the validation set to obtain ValError

IF ValError < MinValError MinValError $=$ ValError $\theta_{\text {opt }}^{-}=\theta^{-}$

\section{ENDIF}

UNTIL ValError $>$ MinValError OR $\theta^{-}=10^{-10}$

Train one RBF-DDA with $\theta_{o p t}^{-}$using the complete training set Test the optimized RBF-DDA on the test set

Fig. 2. Optimizing RBF-DDA through $\theta^{-}$selection 


\section{Experiments}

\subsection{Data Set}

The input variables considered in this work were chosen by an expert (oral surgeon) based on his previous experience. According to the expert, the most important factors which influence the success or failure of a dental implant are those shown in table 1. Some of those factor were also considered in a recent study which used statistical techniques for analyzing dental implant failure [12]. Table 1 shows the input variables together with their possible values in our data set.

Table 1. Input variables

\begin{tabular}{c|c}
\hline Name & Possible values \\
\hline Age (years) & from 17 to 74 \\
\hline Gender & $\{$ male, female $\}$ \\
\hline Implant position & $\begin{array}{c}\{\text { posterior maxilla, anterior maxilla, } \\
\text { posterior mandible, anterior mandible }\}\end{array}$ \\
\hline Implant type & $\{$ conventional, surface treatment $\}$ \\
\hline Surgical technique & $\{$ conventional, complex $\}$ \\
\hline Smoker? & $\{$ yes, no $\}$ \\
\hline $\begin{array}{c}\text { Previous illness } \\
\text { or medical treatment? }\end{array}$ & $\{$ no, yes (diabetes), yes (osteoporosis), yes (radiotherapy) $\}$ \\
\hline
\end{tabular}

The distribution of the dependent variable in our problem is shown in table 2. This is a classification problem with seven classes. One of the classes indicates failure whereas the remaining six classes indicate success, with a variable period of time. Note that this is an imbalanced dataset, since the number of samples per class is quite different.

Table 2. Distribution of dependent variable

\begin{tabular}{l|r|r}
\hline Class & Frequency & Percentage \\
\hline 1 (success - up to 1 year) & 2 & $1.27 \%$ \\
2 (success - from 1 to 2 years) & 24 & $15.29 \%$ \\
3 (success - from 2 to 3 years) & 25 & $15.92 \%$ \\
4 (success - from 3 to 4 years) & 21 & $13.38 \%$ \\
5 (success - from 4 to 5 years) & 16 & $10.19 \%$ \\
6 (success - five years or more) & 62 & $39.49 \%$ \\
7 (failure) & 7 & $4.46 \%$ \\
\hline Total & 157 & $100 \%$ \\
\hline
\end{tabular}




\subsection{Experimental Setup}

Due to the small number of examples in our data set we have used 10-fold crossvalidation in order to compare the machine learning techniques. This is a well known technique widely used to compare classifiers whenever data is scarce [2]. In 10-fold cross-validation the data set is divided in ten disjoints subsets (folds) [2]. Subsequently, the classifier is trained using a data set composed of nine of these subsets and tested using the remaining one. This is carried ten times, always using a different subset for testing. Finally, the cross-validation error is computed as the mean of the ten test errors thus obtained.

In order to improve even more our comparison, we have firstly generated ten versions of our data set by randomly distributing the patterns. Therefore, each data set contains the same patterns yet in different orders. This means that the subsets used in 10-fold cross-validation are different for each random distributed version of our original data set.

We have performed 10-fold cross-validation using each of the ten randomly ordered versions of our data set. Hence, for each classifier, one hundred simulations were carried out (including the training and test phases).

In the case of weighted SVM (WSVM), we have employed the following values for the weights per class: $w_{1}=12, w_{6}=0.3$, and $w_{7}=4$. For the remaining classes, $w_{i}=1$. These values were selected according to the distribution of the samples per class in our dataset, presented in table 2 .

\subsection{Results and Discussion}

In this study we are interested in comparing the machine learning techniques in our problem regarding the classification error and the complexity of the classifiers, that is, the number of training prototypes stored by each of them. The simulations using RBF-DDA with parameter selection [17] were carried out using SNNS [19], whereas SVM and weighted SVM simulations used LIBSVM [6].

Table 3 compares the classifiers with respect to both 10-fold cross-validation errors and the respective number of training prototypes stored by each classifier. Each line of this table shows the 10-fold cross validation error and number of stored prototypes obtained by each classifier using a different version of our data set (with random order of the patterns). The table also presents the mean and standard deviation of the error and of the number of stored prototypes over the ten versions of our data set obtained by each classifier.

The results of table 3 show that SVM and RBF with $\theta^{-}$selection achieved equivalent classification performance (around $24 \%$ mean error). The best results obtained by RBF with $\theta^{-}$selection (shown in table 3) used $\theta^{-}=0.01$. In spite of the similar accuracies obtained, RBF-DDA was able to build considerably smaller classifiers than SVMs in this problem. Hence, in this problem RBF-DDA with $\theta^{-}$selection achieved a better trade-off between accuracy and complexity compared to SVM. RBF-DDA with $\theta^{-}$selection is also much faster to train than SVMs, which can be also an important advantage in practical applications. 
Table 3. Comparison of classifiers: 10-fold cross-validation errors and number of prototypes stored

\begin{tabular}{c|c|c|c}
\hline & $\begin{array}{c}\text { RBF-DDA with } \\
\theta^{-} \text {selection }\end{array}$ & SVM & $\begin{array}{c}\text { weighted SVM } \\
\left(w_{1}=12, w_{6}=0.3, w_{7}=4\right)\end{array}$ \\
\hline Random set 1 & $26.03 \%[73.9]$ & $25.64 \%[111.6]$ & $23.72 \%[109.2]$ \\
Random set 2 & $22.09 \%[73.9]$ & $24.36 \%[101.0]$ & $23.72 \%[98.8]$ \\
Random set 3 & $23.61 \%[73.2]$ & $23.08 \%[108.7]$ & $23.08 \%[107.3]$ \\
Random set 4 & $24.09 \%[73.7]$ & $23.08 \%[102.5]$ & $22.44 \%[99.1]$ \\
Random set 5 & $22.73 \%[73.7]$ & $24.36 \%[106.5]$ & $22.44 \%[104.2]$ \\
Random set 6 & $24.52 \%[73.7]$ & $24.36 \%[101.6]$ & $23.72 \%[103.4]$ \\
Random set 7 & $24.94 \%[73.9]$ & $23.72 \%[101.6]$ & $22.44 \%[98.8]$ \\
Random set 8 & $26.97 \%[73.7]$ & $24.36 \%[97.5]$ & $23.72 \%[95.1]$ \\
Random set 9 & $26.06 \%[73.2]$ & $24.36 \%[107.2]$ & $23.72 \%[102.5]$ \\
Random set 10 & $24.06 \%[73.9]$ & $23.08 \%[102.3]$ & $23.08 \%[103.4]$ \\
\hline \hline mean & $24.51 \%[73.68]$ & $24.04 \%[104.05]$ & $23.21 \%[102.18]$ \\
st.dev & $1.53 \%[0.27]$ & $0.81 \%[4.27]$ & $0.59 \%[4.28]$ \\
\hline
\end{tabular}

The use of WSVM in our problem produced a small improvement in performance compared to both RBF-DDA with $\theta^{-}$selection and SVM, as shown in table 3. WSVM outperformed SVM in our problem and at the same time produced slightly smaller classifiers (table 3 shows that WSVM stored 102.18 prototypes in the mean whereas SVM stored 104.05).

Next, we compared the classifiers regarding both the performance and the number of prototypes stored using Student paired t-test. In order to compare two classifiers using the Student paired t-test, we first perform 10-fold crossvalidation using the same training and test sets for each of the classifiers. Subsequently, we compute the collection of test errors, $\left\{x_{i}\right\}$ for the first classifier and $\left\{y_{i}\right\}$ for the second one. Then, we compute $d_{i}=x_{i}-y_{i}$, which is used to compute $t$ as follows

$$
t=\frac{\bar{d}}{\sqrt{s_{d}^{2} / k}}
$$

where $\bar{d}$ is the mean of $d_{i}, s_{d}$ is the standard deviation of $d_{i}$ and $k$ is the number of folds. In our experiments, we have performed 10-fold cross-validation, thus $k=10$. Moreover, we employ $95 \%$ confidence level. For this confidence level, the t-student distribution table with $k-1=9$ gives $z=2.262$. Hence, for $95 \%$ confidence level, the results produced by two classifiers being compared will be considered statistically different only if $t>z$ or $t<-z$.

The results of hypothesis tests using the Student test with $95 \%$ confidence level for comparing the classifiers regarding classification errors are shown in table 4. Table 5] compares the classifiers regarding the number of prototypes stored. These results were computed from the results shown in table 3 .

Table 4 shows that the difference in performance between the RBF-DDA and SVM classifiers is not statistically significant. Conversely, the results of this 
Table 4. Hypothesis tests for classification errors

\begin{tabular}{c|c|c}
\hline $\begin{array}{c}\text { RBF-DDA }\left(\theta^{-}\right) \text {sel. } \times \\
\text { SVM }\end{array}$ & $\begin{array}{c}\text { RBF-DDA }\left(\theta^{-}\right) \text {sel. } \times \\
\text { Weighted SVM }\end{array}$ & $\begin{array}{c}\text { SVM } \times \\
\text { Weighted SVM }\end{array}$ \\
\hline$t=1.02$ & $t=2.91$ & $t=3.88$ \\
\hline not significant & significant & significant \\
\hline
\end{tabular}

table shows that the differences in performance between RBF-DDA and weighted SVM as well as between SVM and WSVM are statistically significant.

Table 5 shows that the difference in the number of prototypes stored is statistically significant in the three comparisons performed.

Table 5. Hypothesis tests for number of prototypes stored

\begin{tabular}{c|c|c}
\hline $\begin{array}{c}\text { RBF-DDA }\left(\theta^{-}\right) \text {sel. } \times \\
\text { SVM }\end{array}$ & $\begin{array}{c}\text { RBF-DDA }\left(\theta^{-}\right) \text {sel. } \times \\
\text { Weighted SVM }\end{array}$ & $\begin{array}{c}\text { SVM } \times \\
\text { Weighted SVM }\end{array}$ \\
\hline$t=-21.93$ & $t=-20.70$ & $t=3.02$ \\
\hline significant & significant & significant \\
\hline
\end{tabular}

The results obtained in our simulations confirmed an observation that appeared recently in the literature, namely, that SVMs, despite their strong theoretical foundations and excellent generalization performance in a number of problems, are not the best choice for all classification and regression problems 14. Simpler methods such kNN and neural networks can achieve performance comparable to or even better than SVMs in some classification and regression problems 14. In problems, such as the one considered in this paper, where both classifiers obtained the same generalization performance, other performance measures, such as training time, classification time and complexity must be compared. In our case, the simulations showed that RBF-DDA with $\theta^{-}$selection was better in terms of complexity and consequently in terms of classification time as well. RBF-DDA with $\theta^{-}$selection certainly outperforms SVMs concerning training time as well, since we need to select just one parameter whereas with SVMs we need to select two parameters.

\section{Conclusions}

We have presented a comparative study on three machine learning techniques for prediction of success of dental implants. The data set consisted of 157 examples concerning real-world clinical cases. The input variables concerned risk factors for dental implants chosen by an expert (oral surgeon). The simulations were carried out using ten versions of the data set with different random orders of the patterns. For each random data set, the simulations were carried out via 10-fold cross-validation, due to the small size of the data set. The techniques 
compared were support vector machines (SVMs), weighted support vector machines (WSVMs) and RBF-DDA with $\theta^{-}$selection.

The classifiers considered in this study achieved similar classification performance (around $24 \%$ of mean cross-validation error). Yet RBF-DDA with $\theta^{-}$ selection obtained smaller classifiers (73.68 mean number of prototypes) than SVM (104.05 mean number of prototypes) and WSWVM (102.18 mean number of prototypes). This can represent an advantage in practice for RBF-DDA with $\theta^{-}$selection, since the memory requirement and the time to classify novel patterns will be much smaller than those of SVM and WSWM. Nevertheless, WSVM obtained a small improvement in performance (decrease in classfication error around 1\%) compared with RBF-DDA which was statistically significant according to a Student paired t-test with $95 \%$ confidence level.

Future work includes considering other classifiers for this problem such as the multilayer perceptron (MLP) and SVM with other kernel functions as well as evaluating the classification accuracy per class. Another research direction consists in determining the influence of each risk factor (input) on the classification accuracy, such as was done in $[9]$.

\section{References}

1. V. David Sanchez A. Advanced support vector machines and kernel methods. Neurocomputing, 55:5-20, 2003.

2. A.Webb. Statistical Pattern Recognition. Wiley, second edition, 2002.

3. M. Barry, D. Kennedy, K. Keating, and Z. Schauperl. Design of dynamic test equipment for the testing of dental implants. Materials $\&$ Design, 26(3):209-216, 2005 .

4. M. Berthold and J. Diamond. Constructive training of probabilistic neural networks. Neurocomputing, 19:167-183, 1998.

5. Michael R. Berthold and Jay Diamond. Boosting the performance of RBF networks with dynamic decay adjustment. In G. Tesauro et al, editor, Advances in Neural Information Processing, volume 7, pages 521-528. MIT Press, 1995.

6. Chih-Chung Chang and Chih-Jen Lin. LIBSVM: a library for support vector machines, 2001. Software available at http://www.csie.ntu.edu.tw/ ${ }^{c j l i n / l i b s v m . ~}$

7. C. Cortes and V. Vapnik. Support-vector network. Machine Learning, pages 273297, 1995.

8. N. Cristianini and J. Shawe-Taylor. An Introduction to Support Vector Machines. Cambridge University Press, 2000.

9. Dursun Delen, Glenn Walker, and Amit Kadam. Predicting breast cancer survivability: a comparison of three data mining methods. Artificial Intelligence in Medicine, 34(2):113-127, 2005.

10. Federico Girosi Edgar E. Osuna, Robert Freund. Support vector machines: Training and application. Technical Report A.I. Memo 1602, MIT A.I. Lab, 1997.

11. C.-W. Hsu, C.-C. Chang, and C.-J. Lin. A Practical Guide to Support Vector Classification, 2004. Available at http://www.csie.ntu.edu.tw/ ${ }^{\sim c j l i n / l i b s v m . ~}$

12. Donald Hui, J. Hodges, and N. Sandler. Predicting cumulative risk in endosseous dental implant failure. Journal of Oral and Maxillofacial Surgery, 62:40-41, 2004. 
13. P. Laine, A. Salo, R. Kontio, S. Ylijoki, and C. Lindqvist. Failed dental implants clinical, radiological and bacteriological findings in 17 patients. Journal of CranioMaxillofacial Surgery, 33:212-217, 2005.

14. D. Meyer, F. Leisch, and K. Hornik. The support vector machine under test. Neurocomputing, 55:169-186, 2003.

15. A. L. I. Oliveira, B. J. M. Melo, and S. R. L. Meira. Improving constructive training of RBF networks through selective pruning and model selection. Neurocomputing, 64:537-541, 2005.

16. A. L. I. Oliveira, B. J. M. Melo, and S. R. L. Meira. Integrated method for constructive training of radial basis functions networks. Electronics Letters, 41(7):429-430, 2005.

17. A. L. I. Oliveira, F. B. L. Neto, and S. R. L. Meira. Improving RBF-DDA performance on optical character recognition through parameter selection. In Proc. of the 17th International Conference on Pattern Recognition (ICPR'2004), volume 4, pages 625-628. IEEE Computer Society Press, 2004.

18. J. Shawe-Taylor and N. Cristianini. Kernel Methods for Pattern Analysis. Cambridge University Press, 2004.

19. A. Zell. SNNS - Stuttgart Neural Network Simulator, User Manual, Version 4.2. University of Stuttgart and University of Tubingen, 1998. 